\title{
Humanização na atenção básica de saúde na percepção de idosos
}

\section{Humanization in primary health care from the viewpoint of elderly}

\author{
Thaís Jaqueline Vieira de Lima \\ Doutoranda do programa de Pós-graduação em Odontologia \\ Preventiva e Social na Universidade Estadual Paulista Júlio de \\ Mesquita Filho. \\ Endereço: Rua José Bonifácio, 1193, Vila Mendonça, CEP 16015-050, \\ Araçatuba, SP, Brasil. \\ E-mail: thaisodonto®yahoo.com.br

\section{Renato Moreira Arcieri} \\ Livre-docente. Professor Adjunto do Departamento de Odonto- \\ logia Infantil e Social da Universidade Estadual Paulista Júlio de \\ Mesquita Filho. \\ Endereço: Rua José Bonifácio, 1193, Vila Mendonça, CEP 16015-050, \\ Araçatuba, SP, Brasil. \\ E-mail: rarcieriøfoa.unesp.br

\section{Cléa Adas Saliba Garbin} \\ Livre-docente. Professora Adjunto do Departamento de Odonto- \\ logia Infantil e Social da Universidade Estadual Paulista Júlio de \\ Mesquita Filho. \\ Endereço: Rua José Bonifácio, 1193, Vila Mendonça, CEP 16015-050, \\ Araçatuba, SP, Brasil. \\ E-mail: cgarbinळfoa.unesp.br
}

\section{Suzely Adas Saliba Moimaz}

Livre-docente. Professora Titular do Departamento de Odontologia Infantil e Social da Universidade Estadual Paulista Júlio de Mesquita Filho.

Endereço: Rua José Bonifácio, 1193, Vila Mendonça, CEP 16015-050, Araçatuba, SP, Brasil.

E-mail: sasalibaœfoa.unesp.br

\section{Orlando Saliba}

Livre-docente. Professor Titular da Universidade Estadual Paulista Júlio de Mesquita Filho.

Endereço: Rua José Bonifácio, 1193, Vila Mendonça, CEP 16015-050, Araçatuba, SP, Brasil.

E-mail: oasaliba®foa.unesp.br

\section{Resumo}

O objetivo deste estudo foi analisar a percepção do idoso quanto ao cuidado humanizado na atenção básica de saúde, com enfoque sobre os aspectos do atendimento ambulatorial que interferem na qualidade do atendimento. Para isso, realizou-se um estudo do tipo transversal, abrangendo todas as unidades básicas de saúde de um município do interior do Estado de São Paulo, por meio de entrevistas dirigidas aos idosos que frequentavam essas unidades. 0 instrumento utilizado foi desenvolvido pela Organização Mundial da Saúde (OMS), que, em busca de maior objetividade para a avaliação da qualidade do cuidado à saúde, propôs o conceito de responsividade, para referir-se aos elementos não diretamente ligados ao estado de saúde, e sim aos envolvidos no atendimento acolhedor e resolutivo com responsabilidade e vínculo. Dessa forma, os domínios de responsividade avaliados foram: pronta atenção, dignidade, comunicação, autonomia, escolha dos profissionais, confidencialidade e estrutura física e conforto. Os resultados foram analisados através do software Epi Info 3.5.2. Verificou-se que alguns domínios da atenção em saúde, altamente valorizados pelos idosos, tiveram desempenho bem inferior, como: Autonomia, em que apenas 54,4\% tiveram liberdade para tomar decisões sobre sua saúde ou tratamento; e Comunicação, em que 67,6\% não obtiveram informações sobre outros tipos de tratamentos ou exames e $79,2 \%$ não tiveram oportunidade para esclarecimentos quanto às dúvidas sobre o tratamento. Sugere-se, portanto, mudanças nos serviços de atenção básica à saúde prestados, principalmente quando se trata de idosos, uma população especial que necessita receber uma assistência diferenciada.

Palavras-chave: Humanização da assistência, Serviços de saúde, Políticas públicas, Assistência a idosos. 


\section{Abstract}

The objective of this study was to analyze the perception of the elderly population on how humanized is primary health care, focusing on aspects of outpatient services that affect the quality of care. For this purpose, a cross-sectional study was performed, covering all units offering primary health care in a city in the state of São Paulo, by means of interviews targeted at the elderly who attend these units. The instrument used was developed by the World Health Organization (WHO) that proposed the concept of responsiveness searching for greater objectivity in the assessment of healthcare quality. This concept refers to the elements that are not directly linked to the health status, but those involved in the friendly and decisive service with responsibility and bonding. The areas of responsiveness were: prompt attention, dignity, communication, autonomy, option of professionals, confidentiality and physical structure and comfort. The results were analyzed at the Epi Info 3.5.2 software. It was found that some areas of health care, highly valued by older people, had lower performance, for instance: Autonomy, where only $54.4 \%$ were free to make decisions about his/her health or treatment, and Communication, in which $67.6 \%$ did not obtain information on other types of treatments or tests and $79.2 \%$ had no opportunity for clarification regarding questions about their treatments. It is suggested, therefore, that changes should be made in services provided by primary healthcare units, especially when it comes to seniors, a special population that needs to receive a special service.

Keywords: Humanization of Assistance, Health Services, Public Policies, Elderly Care.

\section{Introdução}

Em menos de quarenta anos, o Brasil migrou de um perfil de mortalidade típico de uma população jovem para um quadro caracterizado por enfermidades crônicas e múltiplas, (Gordilho e col., 200o; Veras, 2003), e isto se deve ao exponencial crescimento da população idosa. Stevenson e colaboradores (1997) afirmam que, dentre outros fatores, esse crescimento influencia o modo de gerir a atenção à saúde, visto que uma sociedade em processo de mudanças tem necessidade de adequação dos valores culturais, das políticas sociais e de saúde, de maneira a atender às necessidades e aos problemas decorrentes do envelhecimento populacional.

Diante disso, Saliba e colaboradores (2007) ressaltam a necessidade urgente de formar uma equipe multidisciplinar qualificada com amplo conhecimento de Geriatria e Gerontologia na busca da melhoria da qualidade de vida das pessoas idosas, visto que, ao atender o idoso, a equipe de saúde deve estar atenta a uma série de alterações físicas, psicológicas e sociais que normalmente ocorrem nesses pacientes, e que justificam um cuidado diferenciado. Esses profissionais têm um importante papel com o idoso, pois, acredita-se que, através de uma relação empática, haja uma assistência humanizada e um comprometimento com o cuidado personalizado, garantindo o seu equilíbrio físico e emocional (Franco e col., 1999).

Pelo fato de a humanização em saúde definir-se pelo valor atribuído ao esforço dos sujeitos na produção da saúde, quando se contemplam a autonomia, o protagonismo, a corresponsabilidade e a vinculação das instâncias, põe em evidência (dada a noção de valor) a dimensão da subjetividade e da singularidade. Categorias como vínculo, responsabilidade, autonomia destacam a ênfase na subjetividade, posto que não são categorias do mundo inerte, mas próprias do sujeito. (Reis e col., 2004).

Ao traçar um paralelo entre o cuidar-tratar, Zobolli (2007) cita que o profissional de saúde não deve preocupar-se apenas com o tratar a doença ou aliviar os sinais e sintomas, embora isso faça parte de sua atenção. Mas deve valorizar o cuidar, ou seja, considerar o outro como um fim em si mesmo e não apenas meio para fins científicos, técnicos ou 
institucionais. Isto exige sensibilidade para com as emoções do outro, manifestando interesse, respeito, atenção, compreensão, consideração e afeto, para ser capaz de responder às experiências específicas de aflição e sofrimento trazidas pelas pessoas que buscam a atenção dos profissionais de saúde.

Entretanto, o ambiente insalubre, o regime de turnos, os plantões, os baixos salários, o contato muito próximo com os pacientes, mobilizando emoções e conflitos tornam esses trabalhadores particularmente susceptíveis ao sofrimento psíquico e ao adoecimento devido ao trabalho (Rios, 2008). Somado a isso, a falta de condições técnicas, seja pela carência de capacitação ou materiais torna o atendimento desumanizante pela má qualidade, e consequentemente com baixa resolubilidade (Oliveira e col., 2006), além de desmotivar o profissional para uma mudança de atuação (Simões e col., 2007), pois se a proposta é humanizar o atendimento, não é possível pô-la em prática se aquele que presta o atendimento não estiver em condição para tal.

É o que se vê em muitas das Unidades Básicas de Saúde (UBS), onde os espaços físicos são improvisados e inadequados, afetando negativamente a recepção dos usuários, interferindo na qualidade dos atendimentos, e muitas vezes, impedindo ou impossibilitando, a privacidade dos procedimentos (Simões e col., 2007).

Em virtude desta situação, o Ministério da Saúde criou, no final da década de 1990 o Programa Nacional de Humanização da Assistência Hospitalar (PNHAH), com o objetivo de valorizar a formação educacional dos profissionais de saúde a fim de possibilitar a incorporação de valores e atitudes de respeito à vida humana, consoante à compreensão de que a qualidade em saúde deve ser composta de competência técnica e de interação, não se resumindo somente a aspectos técnicos ou organizacionais, que resultam em progressivo afastamento dos profissionais de saúde dos usuários, diminuindo o vínculo nas relações, tornando-as mais distantes, impessoais e despersonalizadas (Brasil, 2001; Fortes, 2004).

O Programa destaca a importância da conjugação do binômio "tecnologia" e "fator humano e de relacionamento". Há uma divergência entre possuir boas condições de alta tecnologia e nem sempre dispor da delicadeza do cuidado, o que desumaniza a assistên- cia. Por outro lado, se reconhece que não ter recursos tecnológicos, quando estes são necessários, pode ser um fator de estresse e conflito entre profissionais e usuários, igualmente desumanizando o cuidado. Assim, embora se afirme que ambos os itens constituem a qualidade do sistema, o "fator humano" é considerado o mais estratégico pelo documento do PNHAH (Deslandes, 2004).

Assim, a humanização tornou-se uma preocupação dos profissionais de saúde, funcionários e gestores, representando um fator a ser considerado para se ter excelência na qualidade do atendimento em saúde, principalmente em relação aos idosos, devido às condições especiais que apresentam (Lima e col., 2010).

Dessa forma, em 2003, o Ministério da Saúde começou a expandir a humanização além do ambiente hospitalar instituindo a Política Nacional de Humanização da Atenção e Gestão do SUS (HumanizaSUS), cuja meta principal era efetivar os princípios do Sistema Único de Saúde (SUS) nas práticas de atenção e de gestão, tornando-as mais humanizadas e comprometidas com a defesa da vida, fortalecendo o processo de pactuação democrática e coletiva (Brasil, 2004).

É nesse contexto que se vislumbra a implementação de ações e metas constituintes da Política Nacional de Humanização - PNH. Eé no sentido da visibilidade das prioridades, da aplicação de recursos e da obtenção de resultados em contextos variados que a $\mathrm{PNH}$, como política pública, deve-se colocar como objeto de avaliação (Santos-Filho, 2007).

Segundo Donabedian (1984), um dos precursores dos estudos de avaliação da qualidade do cuidado em saúde, a avaliação dessa qualidade pode dar-se sempre em duas dimensões: desempenho técnico, ou seja, aplicação do conhecimento e da tecnologia médica, de modo a maximizar os benefícios e reduzir os riscos; e relacionamento com o paciente. Cabe aqui salientar que o problema já era percebido desde então, e a partir daí inúmeros estudos foram realizados em busca da qualidade da atenção em saúde.

Assim, a satisfação do paciente, o objetivo mais importante nos estudos de avaliação de qualidade, tem sido incluída, geralmente, na avaliação de resultados. No entanto, as pesquisas de satisfação têm sido alvo de numerosas críticas, dentre elas o as- 
pecto subjetivo da categoria "satisfação", que possui diversos determinantes como: grau de expectativa e exigência individuais em relação ao atendimento e características individuais do paciente como idade, gênero, classe social e estado psicológico (Sitzia e Wood, 1997).

Dessa forma, a busca de maior objetividade foi uma das razões para o desenvolvimento do conceito de responsividade, introduzido pela Organização Mundial da Saúde (OMS) no campo da avaliação em saúde, para se referir aos elementos não diretamente ligados ao estado de saúde. Seu fundamento está no pressuposto de que, além de promover e manter a saúde dos indivíduos, o sistema de saúde deve tratá-los com dignidade, facilitar sua participação nas decisões sobre os procedimentos de saúde, incentivar a comunicação clara entre profissional de saúde e usuário e garantir a confidencialidade do histórico médico (Silva, 20oo). É, portanto, uma forma de medir a qualidade e a humanização da assistência à saúde.

Diante do exposto, acredita-se ser importante avaliar a humanização na atenção básica de saúde, a partir de percepções e experiências de idosos, visto que necessitam de um acompanhamento mais frequente e individualizado, em virtude do perfil epidemiológico que apresentam, permeado de patologias crônicas e múltiplas. Além disso, é necessário salientar a escassez de trabalhos que avaliaram a satisfação do usuário focando os aspectos humanísticos do atendimento, principalmente se tratando exclusivamente de idosos, razão fundamental do presente estudo.

\section{Metodologia}

Trata-se de um estudo transversal descritivo, cuja população-alvo foi constituída por idosos, com idade de 60 anos ou mais que buscavam tratamento e cuidados de saúde em Unidades Básicas de Saúde (UBS) de Araçatuba-SP, durante os meses de janeiro e fevereiro de 2009.

Foram incluídos no estudo os idosos que não apresentassem comprometimento mental que impedisse de entender as questões e respondê-las; autorizassem por escrito a entrevista e a divulgação dos dados posteriormente; fossem moradores da cidade e frequentassem alguma UBS do município e não apresentassem perda considerável de audição que interferisse no diálogo com o entrevistador.

Para o cálculo da amostra, considerou-se intervalo de confiança de $95 \%$ e erro relativo previsto de $5 \%$.

Utilizou-se o programa Epi Info 3.5.2 para o cálculo amostral. Levando-se em consideração uma população idosa de 5.337 idosos que foram atendidos em todas as UBS do município nos dois meses que antecederam o estudo, segundo registros da Secretaria de Saúde e Higiene Pública de Araçatuba, obteve-se uma amostra de 360 pessoas.

Utilizou-se como instrumento de coleta de dados um questionário adaptado de um instrumento de medida de responsividade no sistema de saúde, desenvolvido pela OMS denominado Multi-Country Survey Study (MCSS) (Silva e Valentine, 20oo). Este instrumento foi avaliado quanto a sua viabilidade, confiabilidade e validade das questões de responsividade em 41 países, onde foi concluído que as questões deste instrumento são compreendidas e devidamente relatadas entre diversas populações (Valentine e col., 2007).

O questionário mede a responsividade para a atenção hospitalar e ambulatorial, em oito domínios (Valentine e col., 2003) como pode ser visto na Tabela 1.

Dentre os domínios apresentados, o apoio social só é avaliado no atendimento hospitalar, portanto este estudo avaliou apenas sete domínios propostos pela OMS (Valentine e col., 200o; Üstun e col., 2001; WHO, 2005).

Após tradução e adaptação do questionário, ele foi então submetido a um estudo-piloto aplicado em um grupo de pacientes idosos para analisar a aceitação da pesquisa, observar se existia ou não dificuldade de compreensão de alguma ou algumas questões do formulário, e na sequência foram realizadas as devidas correções.

O questionário, que originalmente continha 44 questões, foi adaptado, com base nos resultados do estudo piloto e na relevância das questões para os serviços de saúde no Brasil, passando a apresentar 30 questões, sobre os diversos aspectos do atendimento ambulatorial, como acesso, acolhimento, privacidade, confidencialidade, autonomia, estrutura física e conforto das unidades, relação médico- 
Quadro I - Domínios definidos pelo conceito de responsividade da OMS

\begin{tabular}{|l|l|}
\hline Domínio & Questões Envolvidas \\
\hline Dignidade & Tratamento com respeito e privacidade, sem discriminação ou violência; \\
\hline Autonomia & Participação na tomada de decisões; \\
\hline Confidencialidade & Confidencialidade das informações pessoais; \\
\hline Comunicação & Escuta; tempo suficiente para questionamento; explicações claras; \\
\hline Pronta Atenção & Obtenção de atendimento quando necessário; tempo de espera razoável; \\
\hline Apoio Social & No hospital: visitas, comidas especiais, práticas religiosas; \\
\hline Escolha & Escolha do profissional; \\
\hline Estrutura física e conforto & Limpeza, espaço, ventilação. \\
\hline
\end{tabular}

-paciente, qualidade de atendimento global e geral do tratamento, bem como cuidados relacionados às operações e procedimentos. 0 instrumento foi aplicado por um único entrevistador, sendo as entrevistas individualmente realizadas em todas as UBS do município.

A autorização da Secretaria de Saúde e Higiene Pública do município e a aprovação da administração de cada unidade foram obtidas previamente ao início da pesquisa, além da aprovação pelo Comitê de Ética em Pesquisa da UNESP - Universidade Estadual Paulista, sob processo nํㅜ 2008-00974, aprovado em 19 de agosto de 2008, obedecendo às normas éticas da Resolução 196/96 promulgada pelo Conselho Nacional de Saúde do Ministério da Saúde - Brasil (Brasil, 1996).

Todos os dados foram inseridos e analisados em um software estatístico Epi Info versão 3.5.2 para calcular a distribuição das frequências e porcentagens. Estas foram, em seguida, comparadas aos resultados de pesquisas de outros países, especialmente aqueles que utilizaram o mesmo questionário.

De acordo com a abordagem da OMS no MCSS, os resultados da responsividade foram dicotomizados em boa responsividade (respostas “ótima” e "boa”) e responsividade ruim (respostas "moderada", "ruim" e "péssima”) (WHO, 2006).

\section{Resultados}

A idade média dos 360 idosos entrevistados era 75,1 anos, havendo predomínio do sexo feminino $(61,9 \%)$. Quanto à escolaridade, verificou-se que 6o,6\% tinham frequentado o ensino fundamental, $8,3 \%$ o ensino médio, 1,4\% uma universidade, enquanto 29,7\% não tinham nenhum certificado escolar.

Quanto à renda mensal, a maioria $(62,2 \%)$ vivia com menos de um salário-mínimo, o que evidencia que a maior parte da população idosa que frequenta as UBS públicas do município pertence às camadas sociais menos favorecidas.

$\mathrm{O}$ que mais motivou os idosos a procurarem atendimento na UBS foi a hipertensão ( $52 \%$ ), seguida por diabetes $(21,4 \%)$ e outros $(32 \%)$, que incluem exames gerais e específicos, como check-ups, exame de sangue, de próstata, ginecológico preventivo, etc., doenças cardiovasculares, vacinas e desordens do aparelho respiratório.

A seguir são apresentados os resultados de acordo com os domínios de responsividade avaliados.

\section{Pronta atenção}

Entre os pacientes entrevistados, 93,3\% sempre foram atendidos nas UBS quando necessitaram de cuidados de saúde e buscaram atendimento. Segundo os pacientes que nem sempre conseguiram atendimento $(6,7 \%)$, o principal motivo pelo qual isso aconteceu foi porque tinha muita gente na frente e não deu tempo de atender alguns pacientes $(54,2 \%)$.

A satisfação quanto ao tempo de espera antes do atendimento foi considerada muito boa pela maioria (62\%). No entanto, uma notável parte (137 pacientes $-38 \%$ ) considerou o tempo de espera muito prolongado.

\section{Dignidade}

A maioria dos idosos entrevistados (97,2\%) foram acolhidos e tratados com respeito pelos profissionais de saúde das UBS, no entanto, 2 pacientes 
idosos (o,6\%) afirmaram já terem sido vítimas de discriminação por parte desses profissionais. Aprofundando-se nesse aspecto, ao perguntar ao idoso se já sofreu algum tipo de violência, crueldade ou opressão por parte dos profissionais de saúde da UBS, todos negaram.

Todos os pacientes que realizaram exames e/ou tratamentos tiveram sua privacidade respeitada (98,3\%). O restante dos pacientes $(1,7 \%)$ não fizeram exames nem tratamentos, somente a primeira consulta, portanto essa questão não se aplica a esses pacientes.

\section{Comunicação}

Quando questionados se os profissionais utilizaram uma linguagem fácil de compreender, explicando com clareza as informações sobre sua saúde, tanto da condição atual quanto dos procedimentos que deveriam ser realizados, 89,4\% dos entrevistados afirmaram ter recebido esse tipo de informação.

Porém, a maioria $(67,6 \%)$ não obteve informações sobre outros tipos de tratamentos ou exames que poderiam ser realizados.

Quanto à oportunidade dada aos pacientes para fazer perguntas sobre o seu problema ou para esclarecer dúvidas sobre o tratamento a ser realizado, a maioria afirmou que teve tempo suficiente para esses esclarecimentos $(79,2 \%)$.

\section{Autonomia}

Em relação à liberdade para tomar decisões sobre a saúde ou tratamento, 54,4\% tiveram essa liberdade, porém, não muito distante, $45,6 \%$ dos entrevistados disseram que são os profissionais que decidem o que será feito, sem liberdade alguma para o paciente dar sua opinião e escolher suas preferências.

\section{Escolha dos profissionais}

A maioria dos idosos entrevistados $(61,7 \%)$ teve liberdade de escolher o profissional de saúde ou de escolher uma segunda opinião, se assim desejassem. Diferente dos 138 idosos (38,3\%) que negaram ter essa liberdade, e que são atendidos por "quem" estiver disponível na UBS, sem nenhuma chance de escolha.

\section{Confidencialidade}

Quando questionados se suas informações pessoais foram mantidas em sigilo pelos profissionais de saúde, a maioria (90\%) dos entrevistados acredita que sim. Apenas 9.7\% ficaram na dúvida e afirmaram não saber se os profissionais guardam segredo ou não, e somente 1 paciente (o,3\%) afirmou que suas informações pessoais não foram mantidas em segredo pelos profissionais da UBS que frequenta.

\section{Estrutura física e conforto}

O ambiente físico das UBS, quanto à limpeza, acomodações, espaço suficiente, ventilação, etc., foi classificado pela maioria dos idosos entrevistados como ótimo ou bom (64\%). Entretanto, uma considerável parte dos entrevistados ( $n=130-36 \%$ ) classificou o ambiente físico da UBS que frequenta como moderado, ruim ou péssimo.

\section{Comparação entre a importância atribuída aos domínios de responsividade em relação à expe- riência obtida nas UBS}

O domínio Pronta atenção foi considerado muito importante por $98,1 \%$ dos entrevistados, atribuição semelhante à experiência obtida por $93,3 \%$ dos idosos que sempre foram atendidos quando necessitaram de cuidados. No entanto, no quesito "tempo de espera", apenas $62 \%$ dos idosos afirmaram estar satisfeitos.

Já o domínio Dignidade obteve valores semelhantes tanto na importância dada quanto na experiência obtida, visto ser considerado muito importante no atendimento básico de saúde por 99,7\% dos entrevistados, sendo $97,2 \%$ os idosos que afirmaram terem sido acolhidos e tratados com respeito e $98 \%$ os que tiveram sua privacidade respeitada durante os exames e tratamentos.

O domínio Comunicação na atenção básica de saúde foi considerado muito importante por 99,4\% dos idosos, porém apenas $32,4 \%$ tiveram informações sobre outros tipos de tratamento.

Autonomia, Escolha dos profissionais e Estrutura física e conforto apresentaram as piores avaliações na perspectiva dos idosos quando comparados à importância atribuída e à experiência vivida, diferente do domínio Confidencialidade que apresentou valores elevados tanto em importância quanto na performance, como pode ser visto na figura 3.

Em relação à satisfação geral com o atendimento, apesar de algumas reclamações e críticas, a maioria dos idosos (76\%) classificou este como bom ou muito bom. 
Figura I - Comparação entre a importância e o desempenho do domínio Pronta Atenção. Araçatuba, São Paulo, 2009

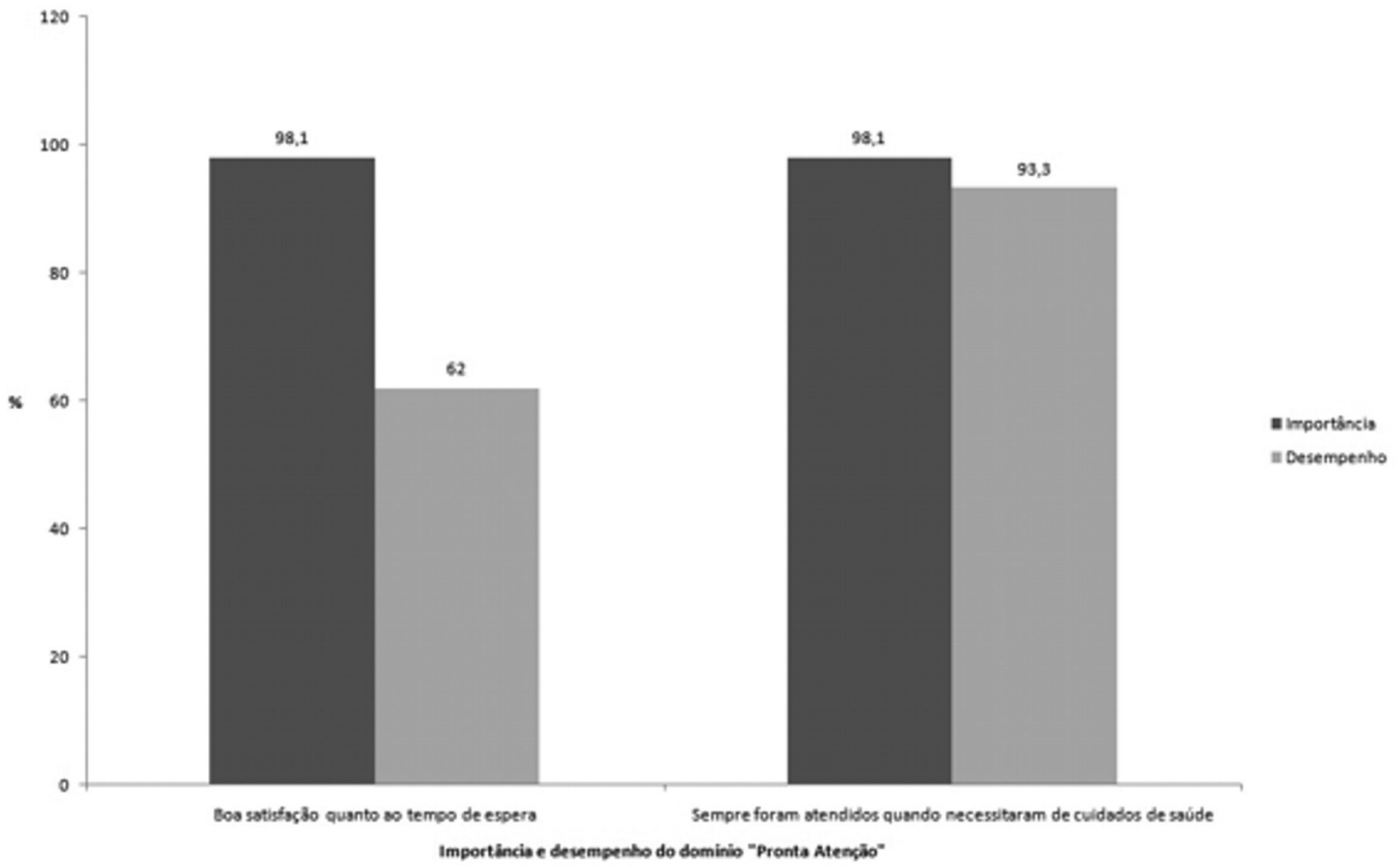

Figura 2 - Comparação entre a importância e o desempenho do domínio Comunicação. Araçatuba, São Paulo, 2009

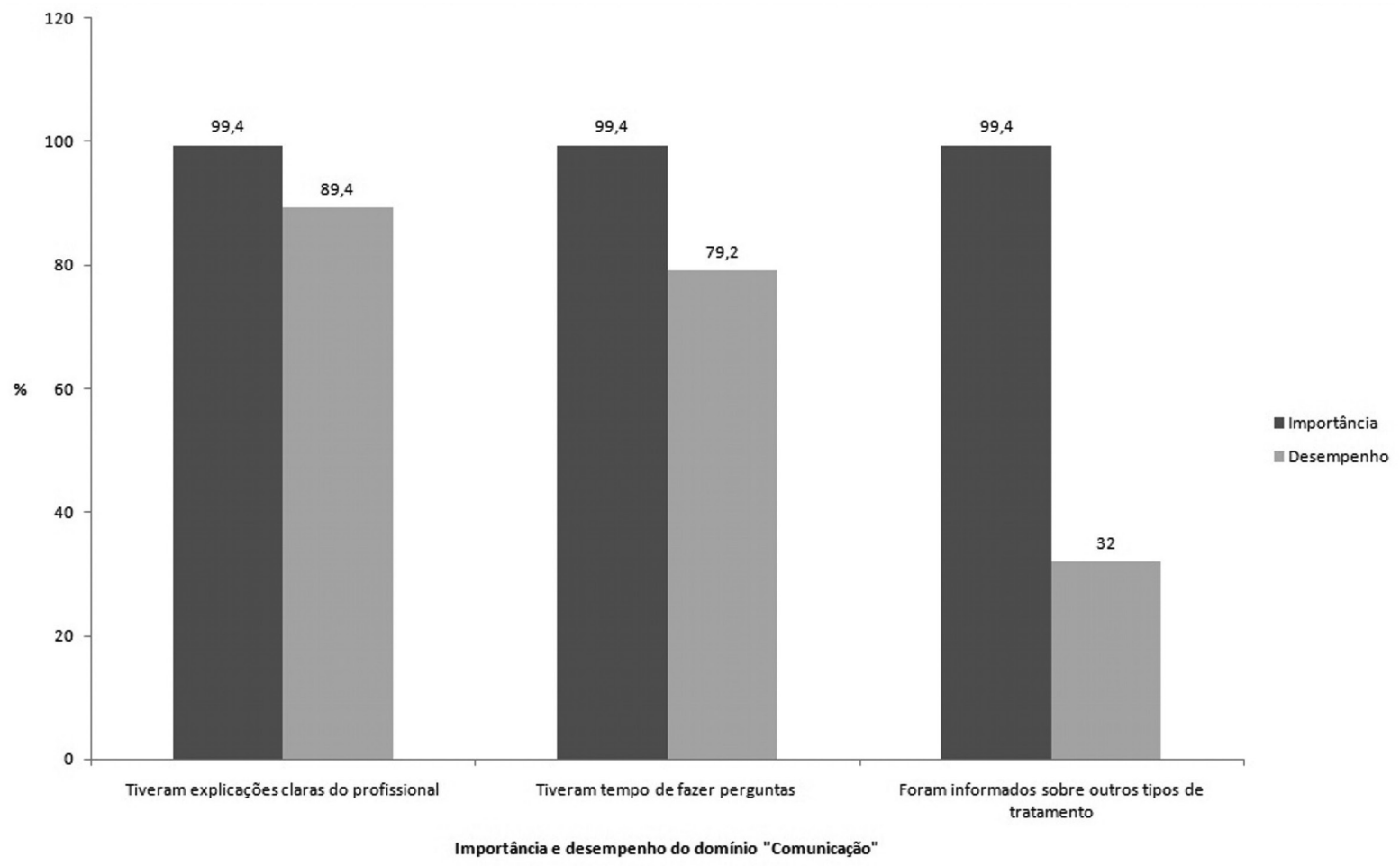


Figura 3 - Comparação entre a importância e o desempenho dos domínios Confidencialidade, Estrutura física e conforto, Escolha dos profissionais, Autonomia. Araçatuba, São Paulo, 2009

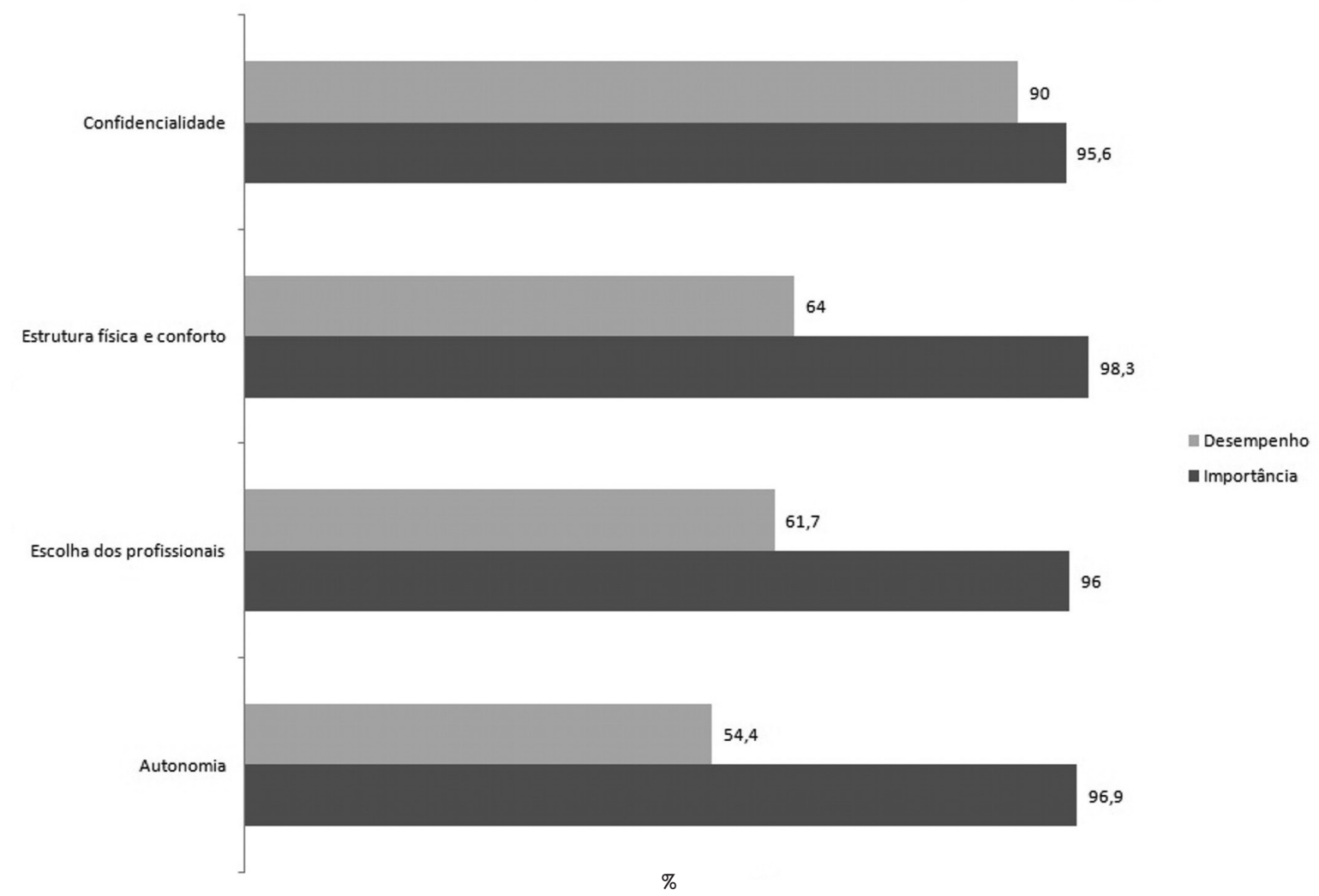

Quanto aos cuidados relacionados às operações e procedimentos, $96,7 \%$ dos idosos acreditam que os profissionais de saúde tiveram habilidade suficiente para tratá-los; $85,8 \%$ afirmaram ter recebido os medicamentos adequados ao seu tratamento e $66,4 \%$ consideraram adequados os equipamentos utilizados pelos profissionais para o seu diagnóstico e/ou tratamento.

Sobre a duração da consulta, $72 \%$ dos idosos entrevistados classificaram-na como ótima ou boa.

\section{Discussão}

Os resultados deste estudo vão além da análise da percepção do idoso quanto aos cuidados humanizados em saúde. Ele traz consigo o retrato das condições da principal porta de entrada para o sistema de atenção à saúde no Brasil, pois as unidades de atenção básica à saúde respondem atualmente por um grande número de consultas médicas além de outros procedimentos. A esse papel assistencial, articulam-se ainda demandas sanitárias, como a vigilância e controle de doenças, bem como riscos de adoecimento, além da educação em saúde. Constituem, portanto, uma forma tecnologicamente específica de atenção que envolve síntese de saberes e complexa integração de ações individuais e coletivas, curativas e preventivas, assistenciais e educativas (Schraiber e col., 1996).

Vê-se aí a grande importância dessas instituições na assistência em saúde, principalmente em se tratando de pessoas que já trazem uma bagagem extensa de enfermidades, em sua maioria crônicas, que sofrem com a falta de assistência e de atividades de lazer, com o abandono em hospitais ou asilos, que vivem angústias com a desvalorização das aposentadorias e pensões, e que sofrem, ainda, todo tipo de obstáculos para assegurar alguma assistência por meio de planos de saúde (Parahyba e Simões, 2006).

De forma geral, o presente trabalho mostra uma boa responsividade na atenção básica de saúde, apesar da detecção de algumas falhas no sistema. 
No entanto, é preciso considerar a observação descrita por Traverso-Yépez e Morais (2004), na qual os autores ressaltam que o usuário do sistema público tem receio de falar mal do atendimento, pois tem medo de "perder a vaga". Somado a isso, observa-se também um conformismo visto através da aceitação implícita da baixa qualidade do serviço público por estar dirigido à população carente, decorrendo daí a “explicação”, a "justificativa” para o descaso verificado pela sua qualidade. Percebe-se assim a "naturalização" de tal situação pelos próprios usuários, que terminam por reconhecer o serviço recebido como um favor ou doação e não como um direito.

Neste trabalho, verificou-se uma discrepância nítida entre a importância dada a determinados aspectos da humanização e ao tratamento recebido nas UBS, como o domínio Pronta atenção, altamente valorizado pelos idosos, e, no entanto, verificou-se uma grande insatisfação com o tempo de espera antes de serem atendidos, fato observado por vários outros autores (Gouveia e col., 2005; Imam e col., 2007; Gomes e col., 2008).

Quanto à Dignidade, a maioria dos entrevistados foi acolhida e tratada com respeito $(97,2 \%)$ sendo que nenhum deles sofreu nenhum tipo de violência, crueldade e opressão por parte dos profissionais, nem tiveram sua privacidade desrespeitada durante os exames e/ou tratamentos, o que corrobora os resultados de vários trabalhos encontrados na literatura (Traverso-Yépez e Morais, 2004; Hsu e col., 2006; Bramesfeld e col., 2007).

Um domínio muito valorizado pelos idosos, e que diz respeito à relação entre médico e paciente é a Comunicação. Observou-se um bom desempenho quanto à oportunidade dada aos pacientes para fazer perguntas, esclarecer dúvidas e receber explicações pelos profissionais, de forma clara e fácil linguagem. Isto também foi observado por Cotta e colaboradores (2005), porém, poucos idosos foram informados sobre outros tipos de tratamentos ou exames que poderiam ser realizados.

Gomes e colaboradores (2008), ao avaliarem a humanização hospitalar por usuários do sistema público de saúde no Brasil verificaram que a comunicação era deficiente, já que o paciente não era tratado pelo nome, nem olhado com interesse, e a linguagem utilizada era incompreensível pela maioria.
Essa divergência entre os resultados do presente estudo com os achados de Gomes e colaboradores (2008) pode ser explicada pela diferença entre as faixas etárias estudadas, o que pode interferir nos resultados de percepção quanto ao atendimento, visto que nosso estudo foi realizado exclusivamente com idosos.

Verificou-se que a maioria dos idosos está satisfeita quanto ao aspecto Comunicação, porém os entrevistados que afirmaram estar insatisfeitos, fizeram graves críticas à forma como foram tratados pelos profissionais de saúde. Com exaltação na voz, vários exclamaram que o profissional nem olhava para suas faces. Relataram que antes de se sentarem na cadeira, o médico já fazia a prescrição medicamentosa e já chamava o próximo, sem ter explicado nada, nem mostrado interesse algum pelo que o paciente estava sentindo.

Em relação à Autonomia, observou-se que pouco mais da metade dos idosos entrevistados afirmaram que tiveram liberdade para tomar decisões sobre sua saúde ou tratamento. Os idosos que não tiveram essa autonomia relataram que quem manda é o médico e que se não obedecessem à sua ordem poderiam até perder a vaga.

Observa-se aí a passividade dos idosos devido ao medo de não serem atendidos. Gomes e colaboradores (2008) constataram os mesmos fatos: uma verdadeira despersonalização e imposição de normas, com desrespeito à autonomia e à decisão do paciente.

Em consonância com Bramesfeld e colaboradores (2007), a Escolha dos profissionais e a Estrutura física e conforto foram os domínios que apresentaram piores avaliações na perspectiva dos idosos, comparadas à importância dada a eles. Notou-se uma grande divergência entre a valoração que os idosos atribuem a esses aspectos em relação ao que receberam nas UBS. A precariedade da estrutura física das unidades também foi relatada por Trad e Esperidião (2010), ao verificarem a percepção de usuários do Nordeste brasileiro.

Apenas Dignidade e Confidencialidade foram bem avaliadas tanto em importância quanto no desempenho, também em conformidade com os resultados encontrados por Bramesfeld e colaboradores (2007). 
Seoane e Fortes (2009), ao investigarem como o usuário do Programa Saúde da Família (PSF) percebe o direito à privacidade e à confidencialidade de suas informações reveladas ao agente comunitário de saúde (ACS), verificaram que existe uma insegurança entre os usuários, de que agravos ou condições de saúde, principalmente as doenças estigmatizadas, como AIDS, tuberculose, câncer, doenças da próstata e o diabetes, fossem mantidas em sigilo. Constataram uma tendência em se admitir que as informações dadas em sigilo podem ser reveladas pelo ACS.

A diferença entre os resultados encontrados por Seoane e Fortes e o presente trabalho pode ser devido ao fato de que os ACS também pertencem à comunidade e, portanto, mantêm um vínculo que extrapola o das relações profissionais, diferentemente dos outros profissionais de saúde. Existiu a necessidade da inclusão de uma categoria que fosse denominada "muito bom, mas com ressalva" para representar todos aqueles depoimentos que, mesmo apontando algum tipo de deficiência, ao serem questionados quanto à satisfação do atendimento de uma forma geral, consideraram este como bom ou muito bom, resultados semelhantes a inúmeros outros estudos (Bailey, 2007; Prato e col., 2007; Marcinowicz e col., 2008). Essa "contradição" subjacente ao discurso de uma parcela significativa dos usuários expressa a complexidade presente na vida cotidiana do serviço de saúde (Traverso-Yépez e Morais, 2004).

Somado a isso, segundo Meireles e colaboradores (2007), o baixo nível de escolaridade deve ser considerado, no planejamento de programas e ações, tanto no âmbito coletivo quanto no individual, pois o nível educacional interfere diretamente no desenvolvimento da consciência sanitária, na capacidade de entendimento do tratamento prescrito e na prática do autocuidado, interferindo diretamente na relação médico-paciente.

Os resultados do presente trabalho demonstram a pertinência em se realizar novos estudos, avaliando a responsividade em outros locais, com grupos de diversas faixas etárias, avaliando não somente a atenção básica, mas também outros programas assistenciais, utilizando a mesma metodologia empregada no presente estudo.

\section{Conclusão}

Com base na metodologia proposta, a humanização na atenção básica de saúde, avaliada a partir de percepções e experiências de idosos, apresentou-se falha em alguns domínios, e essa constatação é essencial para o aprimoramento dos serviços em saúde, visto que oferece um guia para eliminar os problemas em potencial que vêm ocorrendo na assistência básica. Dessa forma, a grande valorização e o baixo desempenho nos domínios Autonomia, Escolha dos profissionais e Estrutura física e conforto sugerem mudanças nos serviços prestados nas Unidades Básicas de Saúde. Para isso, o total envolvimento da equipe de saúde, assim como um pleno engajamento dos gestores e usuários num processo contínuo de avaliação se torna indispensável.

\section{Referências}

BAILEY, V. Satisfaction levels with a community night nursing service. Nursing Standard, London, v. 22, n. 5, p. 35-42, 2007.

BRAMESFELD, A. et al. How does mental health care perform in respect to service user's expectations?: evaluating inpatient and outpatient care in Germany with the WHO responsiveness concept. BMC Health Services Research, London, v. 7, n. 99, p. 1-12, 2007.

BRASIL. Ministério da Saúde. Conselho Nacional de Saúde. Resolução n.196, de 10 de outubro de 1996. Dispõe sobre as diretrizes e normas regulamentadoras de pesquisas envolvendo seres humanos. Diário Oficial [da] República Federativa do Brasil, Brasília, DF, 16 out. 1996.

BRASIL. Ministério da Saúde. Secretaria de Assistência à Saúde. Programa nacional de humanização da assistência hospitalar. Brasília, DF 2001. Disponível em: <http://bvsms.saude.gov. br/bvs/publicacoes/pnhaho1.pdf $>$. Acesso em: 4 abr. 2012.

BRASIL. Ministério da Saúde. HumanizaSUS: política nacional de humanização: a humanização como eixo norteador das práticas de atenção e gestão em todas as instâncias do SUS. Brasília, DF, 2004. 
COTTA, R. M. M. et al. A satisfação dos usuários do programa de saúde da família: avaliando o cuidado em saúde. Scientia Medica, Porto Alegre, v. 15, n. 4, p. 227-234, 2005.

DESLANDES, S. F. Análise do discurso oficial sobre a humanização da assistência hospitalar.

Ciência \& Saúde Coletiva, Rio de Janeiro, v. 9, n. 1, p. 7-14, 2004.

DONABEDIAN, A. La calidad de la atención médica: definición y métodos de evaluación. México: La Prensa Mexicana, 1984.

FORTES, P. A. C. Ética, direitos dos usuários e políticas de humanização da atenção à saúde. Saúde e Sociedade, São Paulo, v. 13, n. 3, p. 30-35, 2004.

FRANCO, T. B.; BUENO, W. S.; MERHY, E. E. O acolhimento e os processos de trabalho em saúde: o caso de Betim, Minas Gerais, Brasil. Cadernos de Saúde Pública, Rio de Janeiro, v. 15, n. 2, p. 345353, 1999.

GOMES, A. M. A. et al. Etno-avaliação da humanização hospitalar pelo usuário do Sistema Único de Saúde (SUS) e seus mediadores. Revista da Escola de Enfermagem da USP, São Paulo, v. 42, n. 4, p. 635-642, 2008.

GORDILHO, A. et al. Desafios a serem enfrentados no terceiro milênio pelo setor saúde na atenção integral ao idoso. Rio de Janeiro: UnATI, 2000.

GOUVEIA, G. C. et al. Health care user's satisfaction in Brazil, 2003. Cadernos de Saúde Pública, Rio de Janeiro, v. 21, S 109-S118, 2005. Suplemento 1.

HSU, C. C. et al. The dimensions of responsiveness of a health system: a Taiwanese perspective. $B M C$ Public Health, London, v. 6, n. 72, p. 1-7, 2006.

IMAM, S. Z. et al. Patient's satisfaction and opinions of their experiences during admission in a tertiary care hospital in Pakistan: a cross sectional study. BMC Health Services Research, London, v. 7, n. 161, p. 1-8, 2007.

LIMA, T. J. V. et al. A humanização na atenção à saúde do idoso. Saúde e Sociedade, São Paulo, v. 19, n. 4, p. 866-877, 2010.
MARCINOWICZ, L.; KONSTANTYNOWICZ, J.; CHLABICZ, S. The patient's view of the acceptability of the primary care in Poland. International Journal For Quality in Health Care, Oxford, v. 20, n. 4, p. 277-283, 2008.

MEIRELES, V. C. et al. Características dos idosos em área de abrangência do programa saúde da família na região Noroeste do Paraná: contribuições para a gestão do cuidado em enfermagem. Saúde e Sociedade, São Paulo, v. 16, n. 1, p. 69-8o, 2007.

OLIVEIRA, B. R. G.; COLLET, N.; VIERA, C. S. A humanização na assistência à saúde. Revista Latinoamericana de Enfermagem, Ribeirão Preto, v. 14, n. 2, p. 277-284, 2006.

PARAHYBA, M. I.; SIMÕES, C. C. S. A prevalência de incapacidade funcional em idosos no Brasil. Ciência \& Saúde Coletiva, Rio de Janeiro, v. 11, n. 4 , p. 967-974, 2006.

PRATO, R. et al. The perception of healthcare quality of elderly in the city of Bari, South Italy. $B M C$ Health Services Research, London, v. 7, n.

174, p. 1-10, 2007.

REIS, A. O. A.; MARAZINA, I. V.; GALLO, P. R. A humanização na saúde como instância libertadora. Saúde e Sociedade, São Paulo, v. 13, n. 3, p. 36-43, 2004.

RIOS, I. C. Humanização e ambiente de trabalho na visão de profissionais da saúde. Saúde e Sociedade, São Paulo, v. 17, n. 4, p. 151-16o, 2008.

SALIBA, N. A., et al. Perfil de cuidadores de idosos e percepção sobre saúde bucal. Interface: Comunicação, Saúde, Educação, Botucatu, v. 11, n. 21, p. 39-50, 2007.

SANTOS-FILHO, S. B. Perspectivas da avaliação na política nacional de humanização em saúde: aspectos conceituais e metodológicos. Ciência \& Saúde Coletiva, Rio de Janeiro, v. 12, n. 4, p. 9991010, 2007.

SCHRAIBER, L. B.; NEMES, M. I. B.; MENDESGONÇALVES, R. B. (Org.). Saúde do adulto: programas e ações na unidade básica. São Paulo: Hucitec, 1996. 
SEOANE, A. F.; FORTES, P. A. C. A percepção do usuário do programa saúde da família sobre a privacidade e a confidencialidade de suas informações. Saúde e Sociedade, São Paulo, v. 18, n. 1, p. 42-49, 2009.

SILVA, A. A framework for measuring responsiveness. Geneva: World Health Organization, 2000.

SILVA, A.; VALENTINE, N. Measuring responsiveness: results of a key informants survey in 35 countries. Geneva: World Health Organization, 2000.

SIMÕES, A. L. A. et al. Humanização na saúde: enfoque na atenção primária. Texto \& Contexto Enfermagem, Florianópolis, v. 16, n. 3, p. 439-444, 2007.

SITZIA, J.; WOOD, N. Patient satisfaction: a review of issues and concepts. Social Science \& Medicine, Oxford, v. 45, n. 12, p. 1829-1843, 1997.

STEVENSON, J. S.; GONÇALVES, L. H. T.; ALVAREZ, A. M. O cuidado e a especificidade da enfermagem geriátrica e gerontológica. Texto $\&$ Contexto-Enfermagem, Florianópolis, v. 6, n. 2, p. 33-55, 1997.

TRAD, L. A. B.; ESPERIDIÃO, M. A. Sentidos e práticas da humanização na estratégia de saúde da família: a visão de usuários em seis municípios do Nordeste. Physis: Revista de Saúde Coletiva, Rio de Janeiro, v. 20, n. 4, p. 1099-1117, 2010.

TRAVERSO-YÉPEZ, M.; MORAIS, N. A.

Reivindicando a subjetividade dos usuários da rede básica de saúde: para uma humanização do atendimento. Cadernos de Saúde Pública, Rio de Janeiro, v. 20, n. 1, p. 80-88, 2004.
ÜSTUN, T. B. et al. WHO multi-country survey study on health and responsiveness: 2000-2001. Geneva: World Health Organization, 2001.

VALENTINE, N. B.; SILVA, A.; MURRAY, C. J. L. Estimating responsiveness level and distribution for 191 countries: methods and results. Geneva: World Health Organization, 2000.

VALENTINE, N. B. et al. Health system responsiveness: concepts, domains and operationalization. In: MURRAY, C. J. L.; EVANS, D. B. (Ed.). Health system performance assessment: debates, methods and empiricism. Geneva: World Health Organization, 2003. p. 573-596.

VALENTINE, N. B.; BONSEL, G. J.; MURRAY, C. J. Measuring quality of health care from the user's perspective in 41 countries: psychometric properties of WHO's questions on health systems responsiveness. Quality of Life Research, Oxford, v. 16, n. 7, p. 1107-1125, 2007.

VERAS, R. Em busca de uma assistência adequada à saúde do idoso: revisão da literatura e aplicação de um instrumento de detecção precoce e de previsibilidade de agravos. Cadernos de Saúde Pública, Rio de Janeiro, v. 19, n. 3, p. 705-715, 2003.

WHO - WORLD HEALTH ORGANIZATION. MCSS data analyst's report, 20o6. Geneva, 2006. Disponível em: <http://www.who.int/ responsiveness/en/>. Acesso em: 4 abr. 2012.

WHO - WORLD HEALTH ORGANIZATION. The health systems responsiveness analytical guidelines for surveys in the multi-country survey study. Geneva, 2005.

ZOBOLLI, E. Ética do cuidado: uma reflexão sobre o cuidado da pessoa idosa na perspectiva do encontro interpessoal. Saúde Coletiva, São Paulo, v. 4 , n. 17, p. 158-162, 2007. 